\title{
Familial Focal Acral Hyperkeratosis Associated with Hyperthyroidism
}

\author{
Ai Akagi, Miki Tanioka ${ }^{1}$ Eri Araki, Kenzo Takahashi, Yoshiki Miyachi and Atushi Utani* \\ Department of Dermatology, Kyoto University Graduate School of Medicine, Kyoto, \\ ${ }^{1}$ Division of Dermatology, Fukui Red Cross Hospital, Fukui, Japan
}

We present a case of familial disease, focal acral hyperkeratosis (FAH), associated with hyperthyroidism that subsequently turned into thiamazole-induced hypothyroidism. To our knowledge, there have been no reports that describe the association between FAH and hyperthyroidism. FAH is a rare type of marginal papular keratoderma and its etiology is still unknown. In the present case, we observed that thiamazoleinduced secondary hypothyroidism was accompanied by a rapid increase of skin lesions. Therefore, we speculate that there is a causal relationship between the deterioration of FAH and the altered thyroid function by the usage of thiamazole.

Keywords: Epidermolytic hyperkeratosis; Hyperthyroidism; Thiamazole

\section{Introduction}

Punctate type of palmoplantar keratoderma is classified into three groups, ${ }^{1}$ including punctate palmoplantar keratoderma (PPPK), filiform keratoderma, and marginal papular keratoderma (MPK). MPKs are a complex group of disorders that share keratotic papules, usually crateriform, along the borders of the hands and feet as a common clinical feature. MPK includes acrokeratoelastoidosis (AKE) and focal acral hyperkeratosis (FAH), which share similar clinical features and histologically identical epidermal alterations. These disorders are distinguished solely by the absence of elastorrhexis in FAH. Here, we present a familial case of FAH associated with hyperthyroidism.

Accepted for Publication: November 10, 2008

*Corresponding author: Atsushi Utani, Department of Dermatology, Kyoto University Graduate School of Medicine, Phone: 81-75-751-3312, FAX: 81-75-751-4949, E-mail: utani@kuhp.kyoto-u.ac.jp

\section{Case Report}

A 57-year-old Japanese woman was referred to our department for evaluation of small asymptomatic firm horny umbilicated papules on the hands and feet. The lesions were first developed at the age of 30 years and gradually increased. These lesions were found on the dorsal surfaces and at the junction between palmar and dorsal skin, especially surfaces of the fingers, metacarpophalangeal and interphalangeal joints (Fig. 1). There were no nail deformities. Two months before her visit, she commenced thiamazole because she was diagnosed as hyperthyroidism with TSH: $0.005 \mu \mathrm{U} / \mathrm{ml}$ (normal: $0.5 \sim 5.0 \mu \mathrm{U} / \mathrm{ml}$ ), free T4 $4.94 \mathrm{ng} / \mathrm{dl}$ (normal: $0.88 \sim 1.62$ $\mathrm{ng} / \mathrm{dl}$ ), free T3 $18.17 \mathrm{pg} / \mathrm{ml}$ (normal: $2.33 \sim 4.00 \mathrm{pg} / \mathrm{ml}$ ). After the treatment, therapy-related hypothyroidism (TSH: 15.1, free T4 0.378, free T3: 2.09) was developed which were accompanied by rapidly increasing skin lesions on her hands. Her past history was not tracable. Her oldest sister and her daughter exhibited 


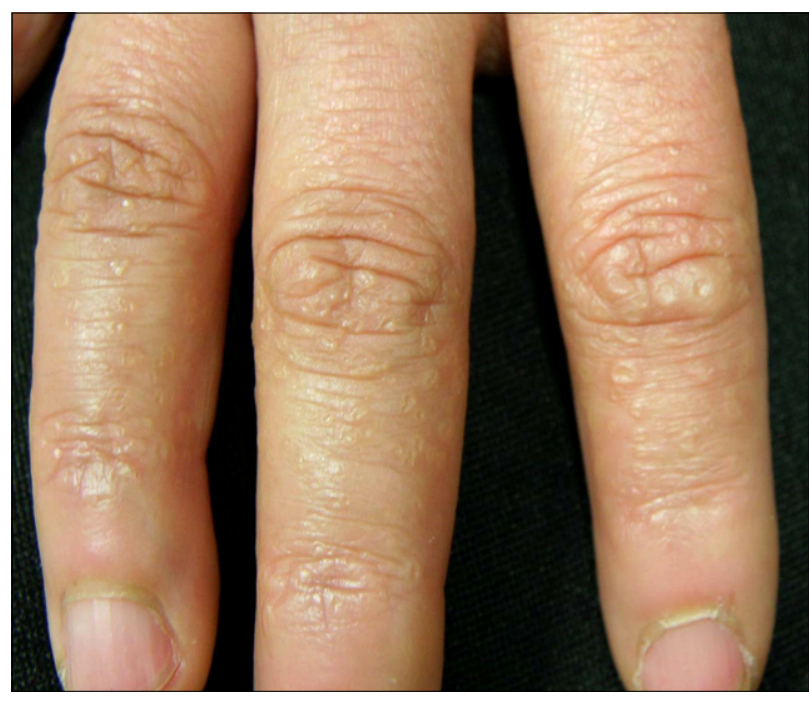

Fig. 1. Multiple, yellow-tinged, flat-topped papules on the fingers,

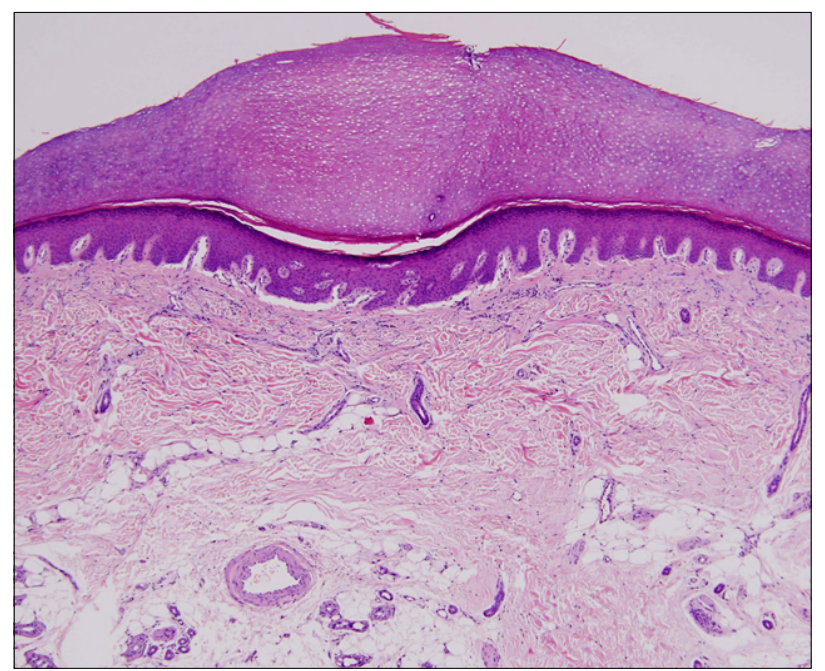

Fig. 2. Pronounced orthohyperkeratosis within a cuplike depression of the epidermis $(H \& E, \times 100)$.

similar skin lesions. Routine laboratory tests were normal except abnormal thyroid hormones. Histopathological examination of skin biopsies from the papules revealed orthohyperkeratosis within focal cup-like depression of the epidermis (Fig. 2). Elastica van Gieson, Weigert, and alcian blue stains showed uniform elastic fibers with no evidence of elastorrhexis. Based on these findings, we diagnosed these papules as FAH. Topical treatment with maxacalcitol showed no response.

\section{Discussion}

AKE and FAH are genodermatoses that share similar clinical features and histologically identical epidermal alteration. FAH is distinguished from AKE on the sole histological basis of lack of elastorrhexis. ${ }^{2-5}$ Both disorders are usually sporadic, although familial cases are autosomal dominant inheritance that have been described like our case and a possible linkage to chromosome 2 has been suggested. ${ }^{4}$ Both form have their onset in childhood or early adult life and frequently develop until the age of 20 years. ${ }^{2,3}$ The lesions are symmetrical and asymptomatic, although unilateral and painful variants have been documented. ${ }^{3}$ Lesions gradually increase in number and size over the years like our case., ${ }^{2,3}$ The patient in our study reported that the number of lesions at the age of 30 years were like that of her 30-year-old daughter.

The causes of AKE and FAH are unknown. ${ }^{2-6}$ No human papilloma virus (HPV) DNA has been detected in affected lesions. There is no evidence that trauma, arsenic ingestion, or light exposure are factors in the development of these disorders. ${ }^{3}$ To our knowledge, there were no reports on an association between $\mathrm{FAH}$ and hyperthyroidism. However, rapid extension of AKE lesions during pregnancy has been reported. ${ }^{3,6}$ In one report, AKE lesions first appeared at the age of 6 years showed rapid extension during her pregnancy at the age of 20 years old. Abnormal thyroid function during pregnancy is well known. In the first trimester of pregnancy, human chorionic gonadotropin (hCG) is rapidly increased at the peak between 7 and 13 weeks of gestation. hCG has mild TSH-like activity, leading to slightly high free T4 and subclinical hyperthyroidism during early pregnancy. At late pregnancy, the level of thyroid hormone becomes relatively low. In our case, thyroid hormones were rapidly decreased after starting thiamazole, accompanying the rapid increase of skin lesions. These findings suggest that thyroid hormone might have a suppressive effect on AKE and FAH. However, two previous reports ${ }^{3,6}$ did not clearly describe AKE deterio- 
rated in late pregnancy. Therefore, the increased lesions of AKE in the present case might depend on the usage of thiamazole rather than altered thyroid functions.

\section{References}

1. Burns T, Breatnach S, Cox N, Griffiths C. Rook's textbook of dermatology. 7th ed. Blackwell Science Ltd., 2004;34-102.
2. Dowd PM, Harman RR, Black MM. Focal acral hyperkeratosis. Br J Dermatol 1983;109:97-103.

3. Highet AS, Rook A, Anderson JR. Acrokeratoelastoidosis. Br J Dermatol 1982;106:337-44.

4. Bogle MA, Hwang LY, Tschen JA. Acrokeratoelastoidosis. J Am Acad Dermatol 2002;47:448-51.

5. Emel E, Mukadder K, Onder B, Pinar A, Ahu B. Focal Acral Huperkeratosis: a rare cutaneous disorder within the spectrum of Costa acrokeratoelastoidosis. Pediatric Dermatology 2004;21:128-30.

6. Nelson-Adesokan P, Mallory SB, Lombardi CL, Lung R. Acrokeratoelastoidosis of Costa. Int J Dermatol 1995;34:431-3. 